\title{
Modelling and robust control of fluidic thrust vectoring and circulation control for unmanned air vehicles
}

D-W Gu*, K Natesan, and I Postlethwaite

Department of Engineering, University of Leicester, Leicester, UK

The manuscript was received on 12 September 2007 and was accepted after revision for publication on 10 December 2007.

DOI: 10.1243/09596518JSCE485

\begin{abstract}
This paper presents actuator models for fluidic thrust vectoring and circulation control and they are used in the design of a robust controller for an unmanned air vehicle. The pitching and rolling moments for the aircraft are produced through the use of a co-flow fluidic thrust vectoring arrangement at the wing trailing edges. Experimental results for the co-flow actuators are used to derive mathematical models and their performance is compared with conventional control surfaces. For the controller design, nonlinear dynamic models are approximated by a simplified linear parameter varying (LPV) model. The polytopic nature of the controller is exploited to reformulate the LPV controller design problem into a $\mu$-synthesis problem. The LPV controllers exhibit superior stability properties over the entire operating region, when compared to conventional gain-scheduling schemes.
\end{abstract}

Keywords: flow control, unmanned air vehicle, linear parameter varying systems, robust control

\section{INTRODUCTION}

Thrust vectoring (TV) has found increasing applications in recent years on aircraft, mostly as an augmentation to conventional control surfaces (see for example [1-3]). Apart from post-stall manoeuvring, advantages of TV include reduced tail area and enhanced stealth, improved low-speed engine-out recoverability, and increased departure resistance [4]. The traditional approach to TV relies on the alteration of the physical boundary conditions of the jet. This is achieved either through external TV, where paddles outside the engine exhaust are used to deflect the exhaust stream, or through internal TV, where a gimballed nozzle is used to deflect the thrust [5]. While traditional TV has found use on various military aircraft, alternate means of TV, namely fluidic thrust vectoring (FTV) and circulation control (CC), have also gained attention in recent years. In contrast to external and internal TV, FTV and CC modify the jet boundary conditions with the reaction surfaces being fixed. Both techniques use the

*Corresponding author: Control and Instrumentation Group, Department of Engineering, University of Leicester, Leicester, UK. email:dag@leicester.ac.uk phenomenon of Coanda jet attachment to convexly curved surfaces to enable either efficient control of a primary jet using a secondary jet (FTV) or to eject a tangential jet over a rounded trailing edge for boundary layer control (CC) [6] (see Figs 1 and 2). Some of the approaches to FTV include the shock thrust vector control method [7] and fluidic throat skewing [8] for supersonic jets, the counterflow technique for both subsonic and supersonic jets [9] and co-flow technique for subsonic jets [10]: the reader is referred to reference [11] for a more detailed account of FTV techniques. The co-flow technique in particular is actively explored for use in

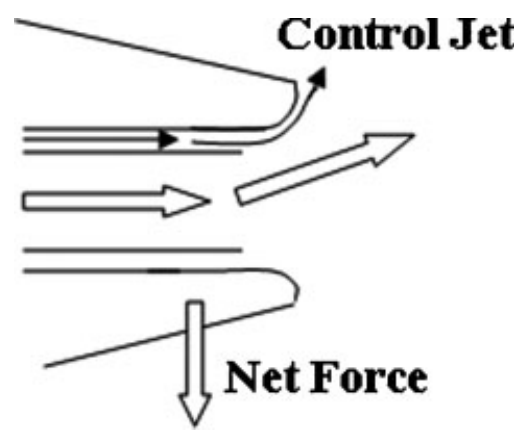

Fig. 1 FTV mechanism 


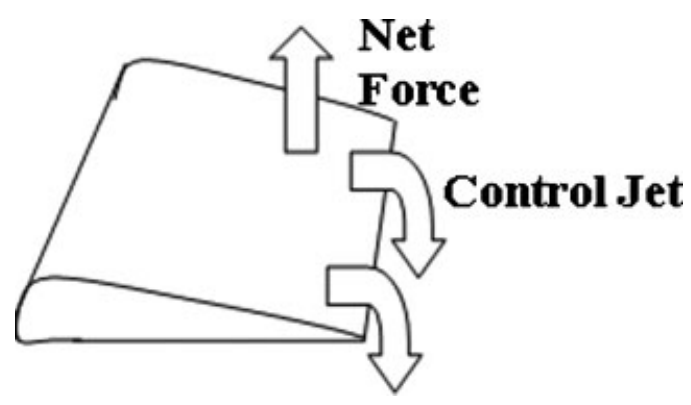

Fig. 2 CC mechanism

subsonic aircraft because of its ease of implementation and effectiveness. This paper attempts to gain insight into the effectiveness of co-flow TV for a subsonic unmanned air vehicle (UAV) through mathematical modelling and robust controller design.

Although fluidic TV is effective, the associated complexity of its dynamics leads to modelling uncertainties. As a result, any controller designed using TV inputs should be robust to modelling uncertainties. In this context, $H_{\infty}$ control and $\mu$ synthesis offer natural means for achieving robust stability and robust performance [12, 13]. Conventional gain-scheduling approaches for $H_{\infty}$ controllers rely on interpolation of individual controllers over the operating region and as a result cannot guarantee stability and performance during transition. In contrast, continuous gain-scheduling, based on an LPV model of the plant, ensures smooth transition and guaranteed stability and performance over the entire-operating region. Over the years, various approaches have been proposed for linear parameter varying (LPV) controller design. In reference [14], a modification of the scaled small-gain theorem is used to find a parameter-varying controller for plants that can be expressed in linear fractional transformation (LFT) form. A single quadratic Lyapunov function that accounts for stability and performance over all possible variations of plant parameters is used in reference [15] for the construction of an LPV controller. The derivation in reference [15] is extended in reference [16] using the bounded real lemma formulation of $H_{\infty}$ performance. Linear fractional representations are also employed in reference [17] for the design of robustly stabilizing controllers using unstructured scaling matrices at different vertices of the parameter polytope. Recently, quadratic LFT Lyapunov functions and full-block multipliers have been used in reference [18] for LPV controller design. The main disadvantage of the above methods is the requirement to solve linear matrix inequality (LMI)s which can be time-consuming. In contrast, the approach presented in this paper uses $\mu$ synthesis for the LPV controller design, with FTV and CC as control inputs. The scheduling parameter (total velocity, as discussed below) is assumed to vary in a polytopic fashion to simplify the design procedure.

An associated issue and not insignificant in the design of LPV controllers is the required LPV modelling of linear plants $[\mathbf{1 9}, \mathbf{2 0}]$. Aircraft dynamics are a function of various parameters, including angle of attack, altitude, and total velocity. However, for the class of UAVs considered in this paper, density changes are negligible and as a result, only total velocity is assumed to vary. In order to make the problem of LPV control more tractable, the LPV modelling in this paper is based on simplified UAV dynamics through approximations in the derivation of stability and control derivatives.

This paper is organized as follows. Section 1 deals with the modelling of FTV and CC actuators for UAVs. A comparison of FTV and CC with conventional control surfaces is presented in section 2 . Section 3 details the design of a robust LPV controller using $\mu$ synthesis. Section 4 gives an analysis of the closed-loop system. Section 5 concludes the paper with the review of the main results.

\section{FTV AND CC MODELLING}

As a part of a BAE Systems/EPSRC programme of research in aeronautical engineering called FLAVIIR (Flapless Air Vehicle Integrated Industrial Research), a demonstrator UAV called DEMON is being built to test, among other things, FTV and CC technologies. Table 1 shows the main physical parameters of the vehicle.

The co-flow FTV technique illustrated in Fig. 1 is used in DEMON, where the tangential injection of secondary air jets around primary jet results in thrust vectoring. Figure 2 shows the CC arrangement where thrust vectoring is achieved through the Coanda jet attachment over curved surfaces. It should be noted that the FTV arrangement described above can be used to achieve moderate thrust vectoring performance only for low subsonic primary exhaust jet Mach numbers. For high primary exhaust jet Mach numbers, supersonic secondary

Table 1 Important physical parameters of DEMON

\begin{tabular}{ll}
\hline Mass & $43.3 \mathrm{~kg}$ \\
Wing span & $2.2 \mathrm{~m}$ \\
Wing mean chord & $1.34 \mathrm{~m}$ \\
Aspect ratio & 2.04 \\
\hline
\end{tabular}


flow is required for adequate control effectiveness [10]. In the case of DEMON, co-flow arrangement is still useful, since the cruise speed range is $22-72 \mathrm{~m} / \mathrm{s}$. While pitching moment is generated through the use of FTV, rolling moment is generated through the asymmetric deflection of primary exhaust jet along the trailing edges of the wing through CC. Rudder is used for producing yawing moment. More details about the thrust vectoring arrangements for DEMON can be found in reference [21].

The general equations of motion for the longitudinal dynamics of DEMON are given by

$$
\begin{aligned}
{\left[\begin{array}{c}
\dot{u} \\
\dot{w} \\
\dot{\theta} \\
\dot{q}
\end{array}\right]=} & {\left[\begin{array}{cccc}
X_{u} & X_{w} & -g \cos \left(\theta_{1}\right) & X_{q} \\
Z_{u} & Z_{w} & Z_{\theta} & Z_{q} \\
0 & 0 & 0 & 1 \\
M_{u} & M_{w} & M_{\theta} & M_{q}
\end{array}\right]\left[\begin{array}{c}
u \\
w \\
\theta \\
q
\end{array}\right] } \\
& +\left[\begin{array}{c}
X_{\delta \mathrm{f}} \\
Z_{\delta \mathrm{f}} \\
0 \\
M_{\delta \mathrm{f}}
\end{array}\right]\left[\delta_{\mathrm{f}}\right]
\end{aligned}
$$

where $u$ is the velocity along the $x$-axis of the bodyaxes coordinate system, $w$ is the velocity along the $z$ axis of the body-axes coordinate system, $\theta$ is the pitch attitude, $q$ is the pitch rate, and $\delta_{\mathrm{f}}$ is the thrust vector angle. $X_{\delta \mathrm{f}}, Z_{\delta \mathrm{f}}$, and $M_{\delta \mathrm{f}}$ are the axial force, normal force, and pitching moment control coefficients with respect to the thrust vector angle that will be derived in this paper.

Let $\dot{M}_{\mathrm{s}}$ be the rate of secondary jet momentum flowrate and $F_{z}$ be the normal force developed by the nozzle. Then $F_{z}$ is related to $\dot{M}_{\mathrm{s}}$ through a simple gain term: $F_{z}=G_{\mathrm{TV}} \dot{M}_{\mathrm{s}}$. The constant of proportionality, $G_{\mathrm{TV}}$, is found from experiments to be $\approx 3[\mathbf{2 1}]$. The blowing coefficient $C_{\mu}$ is defined as the ratio of secondary jet momentum to total jet momentum. Thus

$$
C_{\mu}=\frac{\dot{M}_{\mathrm{s}}}{\dot{M}_{\mathrm{s}}+\dot{M}_{\mathrm{p}}}
$$

where $\dot{M}_{\mathrm{p}}$ is the flowrate of primary exhaust jet momentum. The thrust vectoring angle is then proportional to the blowing coefficient through the constant of proportionality $G_{\mathrm{TV}}, \delta_{\mathrm{TV}}=G_{\mathrm{TV}} C_{\mu}$. Note that $\dot{M}_{\mathrm{p}}$ is a function of throttle setting. Thus the thrust vectoring angle can be obtained from the blowing coefficient, and vice versa, through a simple gain factor. The normalized force coefficients, denoted by $C_{F i}$, where $i=x, y, z$ corresponding to the $X, Y$, and $Z$ axes in the body-axes coordinate system are given by

$$
C_{F i}=\frac{F_{i}}{\dot{M}_{\mathrm{s}}+\dot{M}_{\mathrm{p}}}
$$

Figure 3 shows the variation of $C_{F x}$ and $C_{F z}$ with blowing coefficient, obtained from experiments [21]. As the results are expressed in terms of force coefficients, it is important to convert them into forces along their respective axes, i.e. the $C_{F i}$ are converted into $F_{i}$ in order to use them in the control derivative form in equation (1). From equations (2) and (3), it is obvious that either $\dot{M}_{\mathrm{p}}$ or $\dot{M}_{\mathrm{s}}$ is required to calculate $F_{i}$. The primary jet momentum flowrate is given as

$$
\dot{M}_{\mathrm{p}}=p_{\mathrm{atm}} A M_{\mathrm{p}}^{2} \gamma
$$

where $p_{\text {atm }}$ is the atmospheric pressure, $A$ is the nozzle cross-sectional area, and $\gamma$ is the ratio of specific heats. The experiments were conducted at an exhaust jet temperature of $600^{\circ} \mathrm{C}$ and values of $M_{\mathrm{p}}$ (primary jet Mach number) ranging from 0.14 to 0.33 . Thus the value of $\gamma$ is 1.346 . For values of $p_{\text {atm }}=101325 \mathrm{~Pa}, A=0.0012 \mathrm{~m}^{2}$, and for an average value of $M_{\mathrm{p}}=0.226$ it is found that $\dot{M}_{\mathrm{p}}=30.84 \mathrm{~N}$.

From the plots, and using the results of the preceding discussion, the variations of $F_{x}$ and $F_{z}$ with respect to $\delta_{\mathrm{f}}$ are found to be $F_{x \delta_{\mathrm{f}}}=$ $-0.03166 \mathrm{~N} / \mathrm{deg}, F_{z \delta_{\mathrm{f}}}=-0.01516 \mathrm{~N} / \mathrm{deg}$. The control derivatives in equation (1) are then obtained from force derivatives using the following formulae

$$
X_{\delta \mathrm{f}}=\frac{\rho_{1000} V^{2} F_{x \delta_{\mathrm{f}}}}{m \rho V_{\mathrm{e}}^{2}} \text { and } Z_{\delta \mathrm{f}}=\frac{\rho_{1000} V^{2} F_{z \delta_{\mathrm{f}}}}{m \rho V_{\mathrm{e}}^{2}}
$$

where $\rho_{1000}$ is the density at $1000 \mathrm{~m}$, the altitude at which the aircraft is assumed to cruise, $V$ is the trim velocity at which the control derivatives are evaluated, $m$ is the mass of the aircraft, and $\rho$ is the density on the ground, where the experiments were conducted. $V_{\mathrm{e}}$ is the velocity at the experimental conditions, $V_{\mathrm{e}}=76.9 \mathrm{~m} / \mathrm{s}$. At $V=22 \mathrm{~m} / \mathrm{s}$, the value of $X_{\delta \mathrm{f}}=-0.0035 \mathrm{~m} \mathrm{~s}^{-2} / \mathrm{rad}$, while that of $Z_{\delta \mathrm{f}}=$ $-0.00162 \mathrm{~m} \mathrm{~s}^{-2} / \mathrm{rad}$. The moment arm from the FTV application point to the centre of gravity of the aircraft is 96.2 per cent of the mean geometric chord, or $1.29 \mathrm{~m}$. Using this value of moment arm, the moment derivative is calculated to be $M_{\delta \mathrm{f}}=$ $-0.000612 \mathrm{~s}^{-2} / \mathrm{rad}$.

The lateral dynamics of aircraft with CC are represented as 

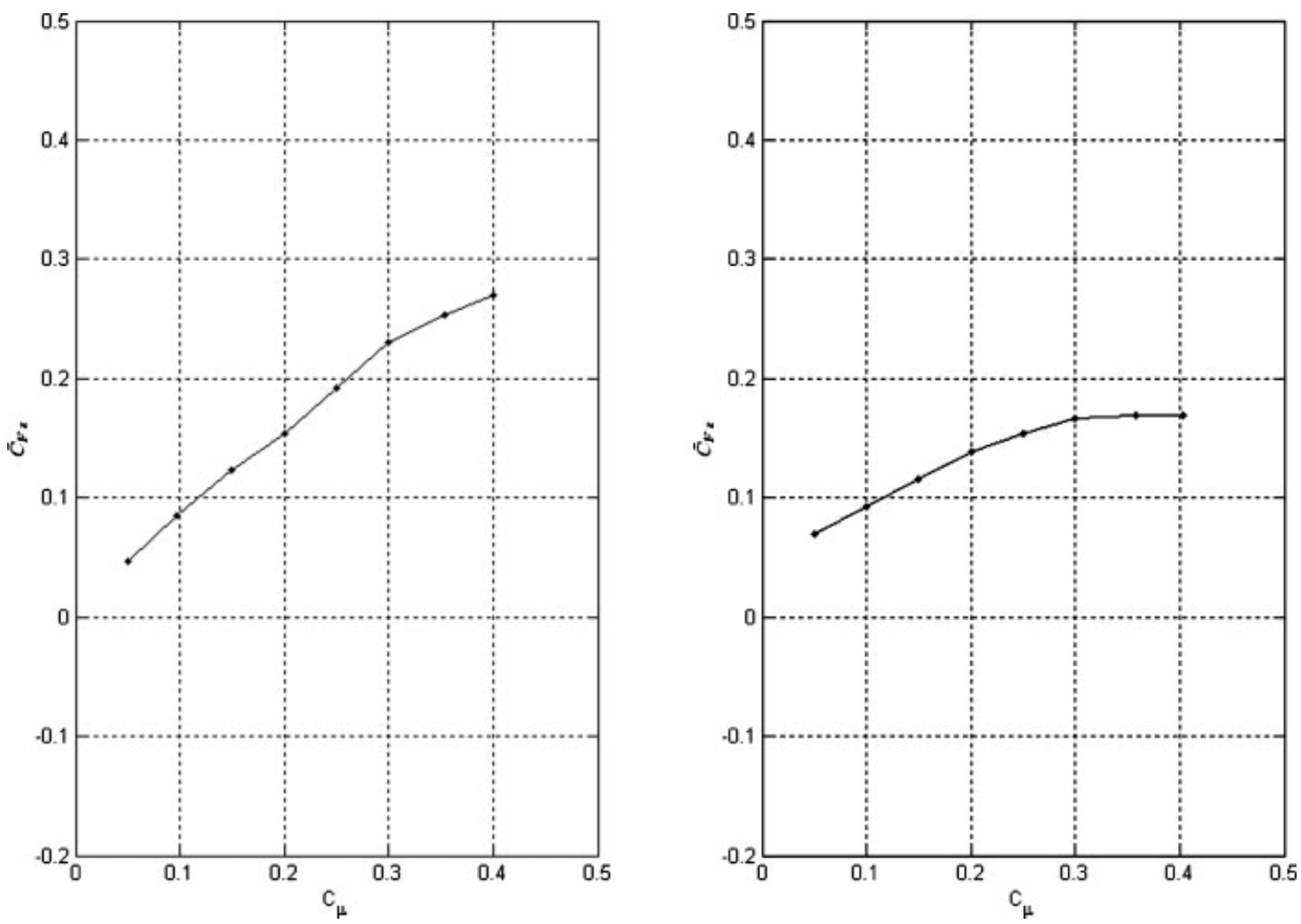

Fig. 3 Variation of $C_{F x}$ with (a) $C_{\mu}$ and (b) with thrust vector angle

$$
\left[\begin{array}{c}
\dot{v} \\
\dot{p} \\
\dot{r} \\
\dot{\phi}
\end{array}\right]=\left[\begin{array}{cccc}
y_{v} & y_{p} & y_{r} & y_{\phi} \\
l_{v} & l_{p} & l_{r} & l_{\phi} \\
n_{v} & n_{p} & n_{r} & n_{\phi} \\
0 & 1 & 0 & 0
\end{array}\right]\left[\begin{array}{c}
v \\
p \\
r \\
\phi
\end{array}\right]+\left[\begin{array}{c}
y_{\delta \mathrm{c}} \\
l_{\delta \mathrm{c}} \\
n_{\delta \mathrm{c}} \\
0
\end{array}\right]\left[\delta_{\mathrm{c}}\right]
$$

where $v$ is the perturbed velocity along the body $y$ axis, $p$ is the roll rate, $r$ is the yaw rate, and $\varphi$ is the roll angle. $\delta_{\mathrm{c}}$ is the deflection of the CC jet. The sideforce coefficient $y_{\delta c}$ is negligible, as the major source of side-force is the rudder. Hence, this paper will not detail the derivation of $y_{\delta c}$. Again, data from experimental results are used for the calculation of the other control derivatives. Figure 4 shows the variation of the rolling moment coefficient, $C_{\mathrm{l}}$ with the blowing coefficient at different angles of attack. The value of $l_{\delta \mathrm{c}}$ is then calculated to be 5.3.

A similar approach is used for the calculation of the yawing moment coefficient and side-force coefficient. Figure 5 shows the variation of the yawing moment coefficient $C_{n}$ with $c_{\mu}$ at different angles of attack, as determined from experiments. The highly non-linear dependence of the yawing moment coefficient on the blowing coefficient means that the value of $c_{n \mu}$ changes with both angle of attack and blowing coefficient. However, approximating the slope around the nominal blowing coefficient of 0.0005 , for small deflections, it is found that the average value of $c_{n \mu}$ is 0.0083 or $l_{\delta \mathrm{c}}=0.7149$.

\section{PERFORMANCE COMPARISON OF FTV AND CC ACTUATORS WITH CONVENTIONAL CONTROL SURFACES}

The preceding section explained the means of producing pitching and rolling moments using coflow techniques and also presented the calculation of control derivatives. However, it is important to know the characteristics of FTV performance in comparison with those of conventional elevators and ailerons in order to design an effective flight control system. Figure 6 shows a comparison of the $Z$-force derivative with respect to the DEMON's elevator and FTV at different trim speeds. As can be seen, the variation of lift with unit deflection of elevator is higher than that caused by thrust vectoring. The disparity in absolute magnitude increases with trim velocity $\left(Z_{\delta \mathrm{e}}=-0.0566 \mathrm{~m} \mathrm{~s}^{-2} / \mathrm{rad}\right.$ and $Z_{\delta \mathrm{f}}=$ $-0.00162 \mathrm{~ms}^{-2} / \mathrm{rad}$ at $22 \mathrm{~m} / \mathrm{s}$, while $Z_{\delta \mathrm{e}}=$ $-0.5800 \mathrm{~m} \mathrm{~s}^{-2} / \mathrm{rad}$ and $Z_{\delta \mathrm{f}}=-0.1888 \mathrm{~m} \mathrm{~s}^{-2} / \mathrm{rad}$ at $72 \mathrm{~m} / \mathrm{s}$ ). Figure 6 also shows the comparison of the pitching moment derivative with respect to the 


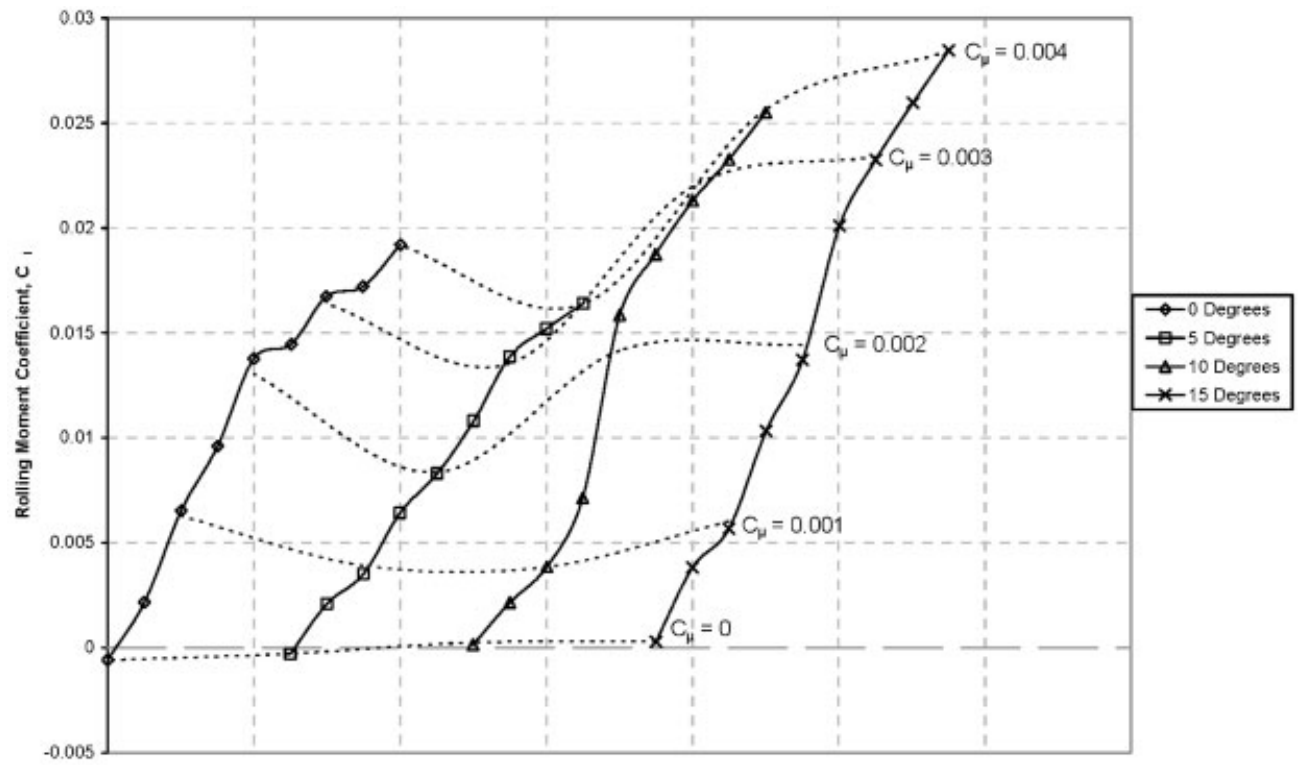

Fig. 4 Variation of $c_{1}$ with $c_{\mu}$ at different angles of attack

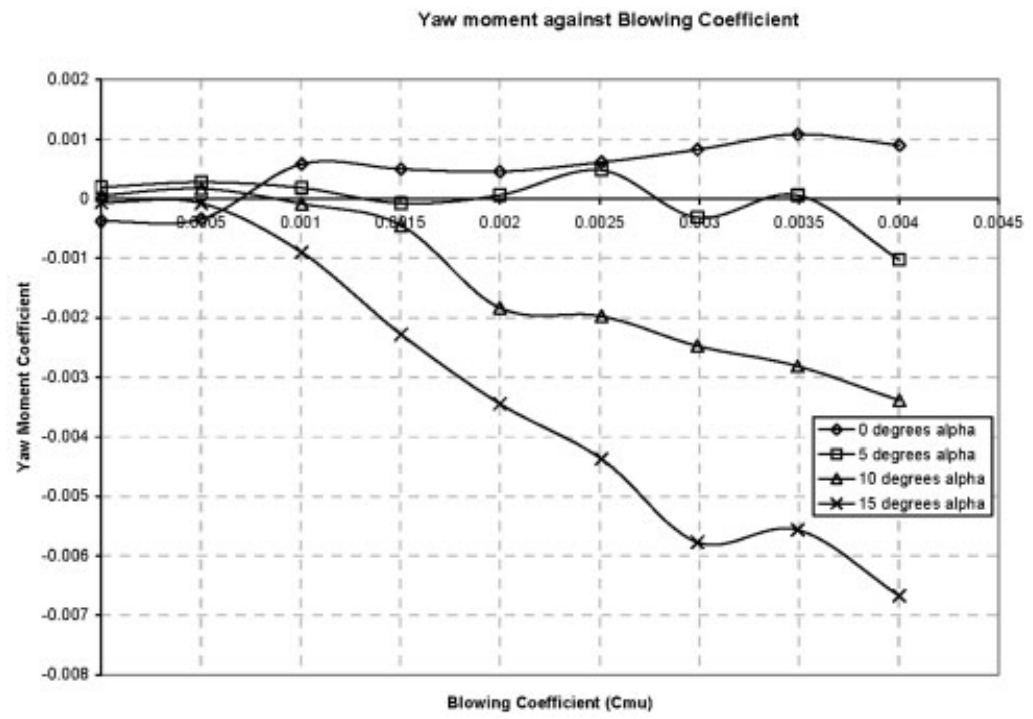

Fig. 5 Variation of $c_{n}$ with $c_{\mu}$ at different angles of attack

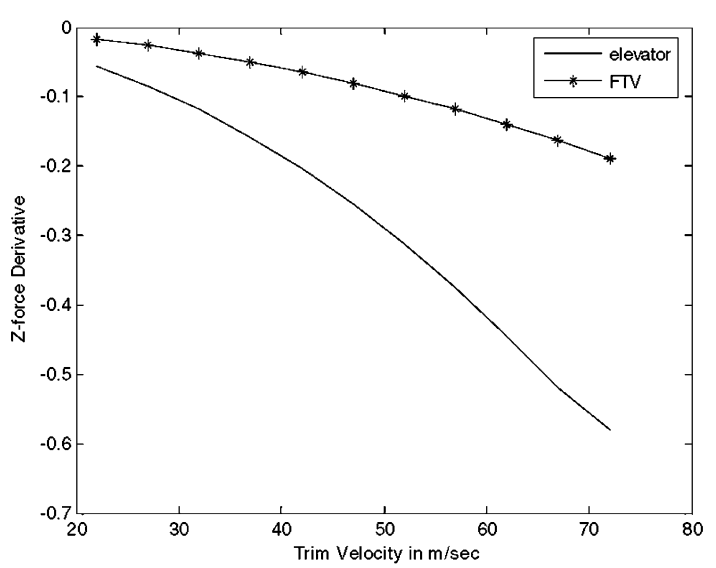

(a)

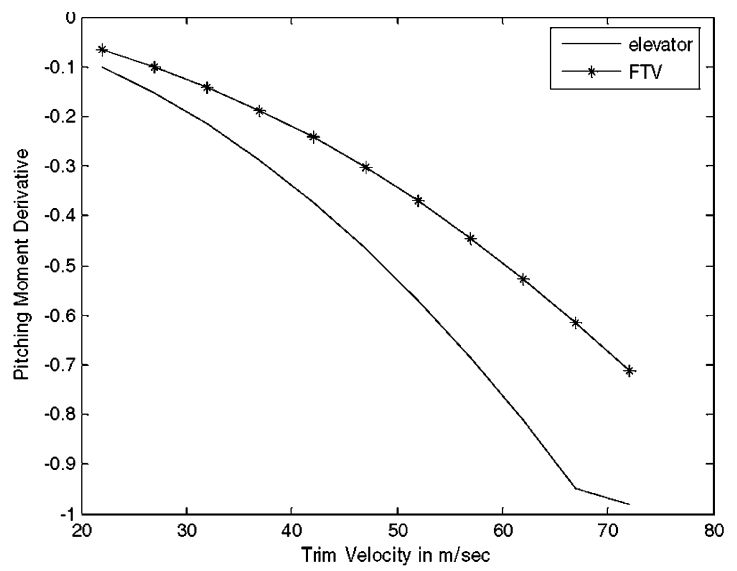

(b)

Fig. 6 (a) $Z$-force and (b) pitching moment derivatives as functions of trim velocity 
elevator and FTV. Again, the pitching moment derivative owing to the elevator is higher than that owing to FTV $\left(M_{\delta \mathrm{f}}=-0.0663 \mathrm{~s}^{-2} / \mathrm{rad}\right.$ and $M_{\delta \mathrm{e}}=$ $-0.0998 \mathrm{~s}^{-2} / \mathrm{rad}$ at $22 \mathrm{~m} / \mathrm{s}$ and $M_{\delta \mathrm{f}}=-0.7106 \mathrm{~s}^{-2} /$ $\operatorname{rad}$ and $M_{\delta \mathrm{e}}=-0.98 \mathrm{~s}^{-2} / \mathrm{rad}$ at $72 \mathrm{~m} / \mathrm{s}$ ). Thus, the control effort (primary jet deflection) required by FTV is approximately 30 per cent more than the deflection required by the elevator. In other words, if a powerful and reliable source of FTV can be provided onboard, the FTV can replace the elevator as a basic control surface, albeit with some loss in effectiveness.

A comparison of rolling moment derivatives and yawing moment derivatives, as shown in Fig. 7 also reveals that the CC performance is poorer than the DEMON's aileron. More precisely, averaging over the cruise speed range of 22 to $72 \mathrm{~m} / \mathrm{s}$, CC is 27 per cent less effective than the aileron in the generation of rolling moments and for yawing moments CC is 50 per cent less effective. An important fact to note is that the ratio of yawing moment to rolling moment from CC is 10 per cent, whereas it is 16 per cent for the aileron. This is advantageous since the aircraft yaws against the turn and smaller the yawing moment, smaller the rudder correction required.

\section{DESIGN OF A ROBUST LONGITUDINAL CONTROLLER WITH FTV}

This section presents the design of LPV controllers for DEMON. Owing to space limitations, only the detailed flight control system design for the longitudinal dynamics is presented. Lateral flight control system design with CC follows similarly. Figure 8 shows the closed-loop configuration for longitudinal controller design. $r=\left[\begin{array}{c}h \\ V_{\mathrm{T}}\end{array}\right]$ is the reference signal, where $h$ is the altitude and $V_{\mathrm{T}}$ is the total velocity.

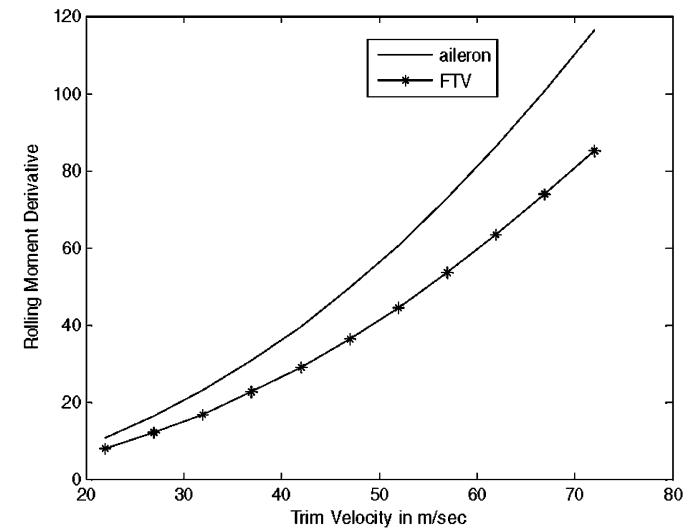

(a)

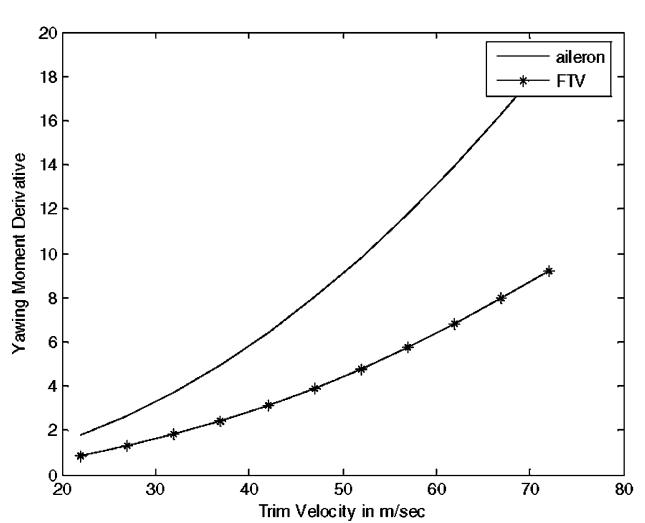

(b)

Fig. 7 (a) Rolling moment and (b) yawing moment derivatives as functions of trim velocity

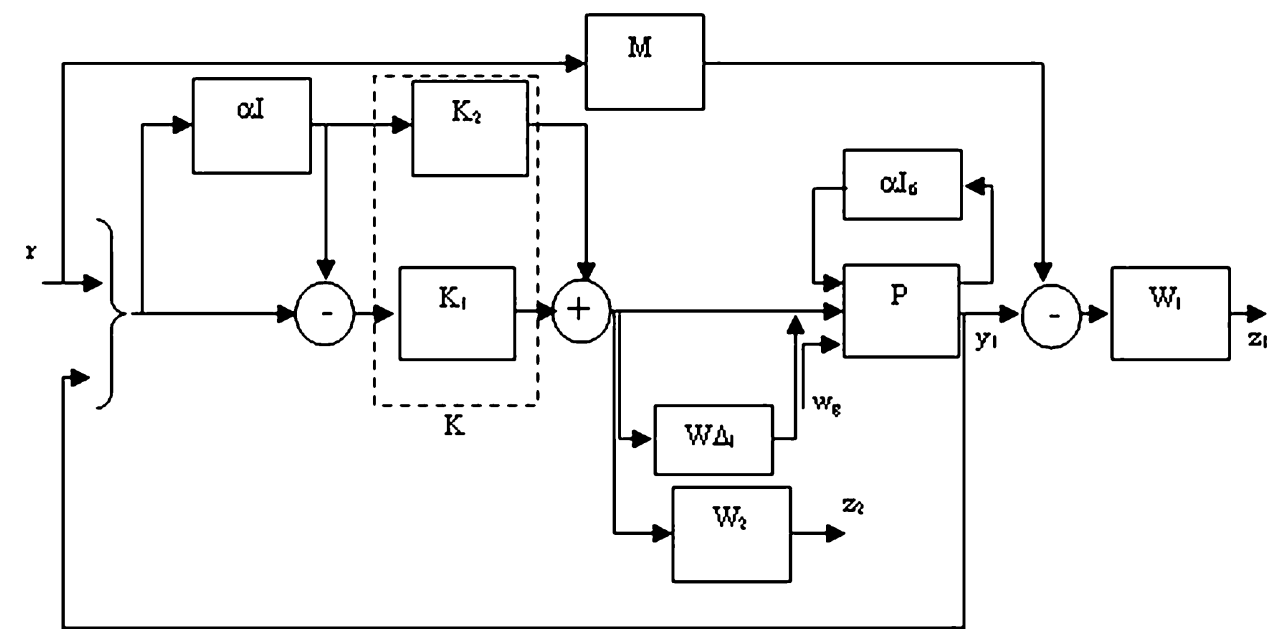

Fig. 8 Two-degree-of-freedom model matching configuration 
The idea is to force the aircraft to follow altitude and velocity reference commands.

$P$ is the LPV plant with the state-space realization $P=\left[\begin{array}{cc}A(V) & B \\ C & 0\end{array}\right]$. It comprises the LPV longitudinal model and a set of high-frequency actuators and sensors at the inputs and outputs respectively. The addition of sensors and actuators helps in confining the LPV dependence of the plant to the system matrix ' $A$ '. LPV model of the longitudinal dynamics is represented as

$$
\begin{gathered}
{\left[\begin{array}{c}
\dot{u} \\
\dot{w} \\
\dot{\theta} \\
\dot{q}
\end{array}\right]=\left[\begin{array}{cccc}
X_{u}(\mathrm{~V}) & X_{w}(\mathrm{~V}) & -g \cos \left(\theta_{1}(\mathrm{~V})\right) & X_{q}(\mathrm{~V}) \\
Z_{u}(\mathrm{~V}) & Z_{w}(\mathrm{~V}) & Z_{\theta}(\mathrm{V}) & Z_{q}(\mathrm{~V}) \\
0 & 0 & 0 & 1 \\
M_{u}(\mathrm{~V}) & M_{w}(\mathrm{~V}) & M_{\theta}(\mathrm{V}) & M_{q}(\mathrm{~V})
\end{array}\right]} \\
{\left[\begin{array}{c}
u \\
w \\
\theta \\
q
\end{array}\right]+\left[\begin{array}{cc}
X_{\delta \mathrm{f}}(V) & X_{\delta \mathrm{t}}(V) \\
Z_{\delta \mathrm{f}}(V) & Z_{\delta \mathrm{t}}(V) \\
0 & 0 \\
M_{\delta \mathrm{f}}(V) & M_{\delta \mathrm{t}}(V)
\end{array}\right]\left[\begin{array}{l}
\delta_{\mathrm{f}} \\
\delta_{\mathrm{t}}
\end{array}\right]}
\end{gathered}
$$

where $\delta_{\mathrm{f}}$ is the FTV deflection and $\delta_{\mathrm{t}}$ is the throttle setting. The stability and control derivatives in equation (6) are functions of trim velocity and are found through the method of least-squares curve fit. While the derivatives vary in both linear and quadratic fashion as a function of velocity, only the
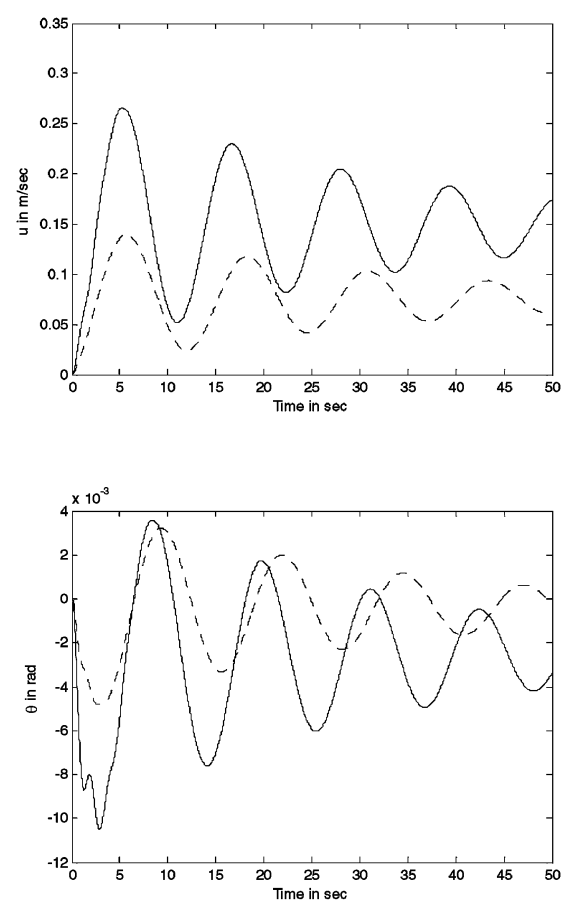

Fig. 9 Open-loop time responses of actual model and LPV model at $22 \mathrm{~m} / \mathrm{s}$

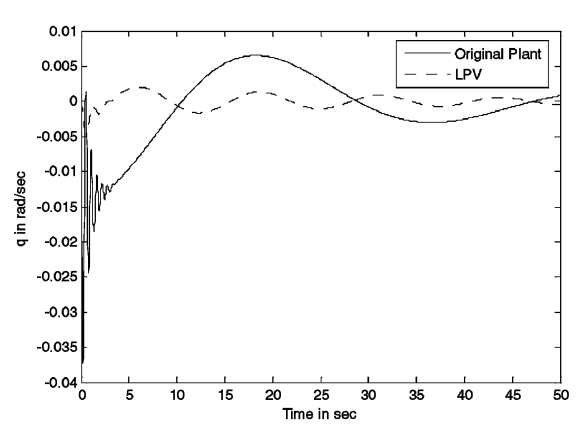

linear dependence is used to make the problem simple and tractable. Also, the coefficients that most influence the dynamics are found by fixing all other coefficients in equation (6) at their average values, while in turn varying one coefficient over its entire range. The frequency and time responses of the model can then be analysed at various trim velocities to determine how a particular coefficient influences the dynamic characteristics. Proceeding in this way, it is determined that the coefficients $X_{q}, Z_{\theta}, Z_{q}, M_{u}$, $Z_{\delta \text { e }}$, and $M_{\delta \mathrm{e}}$ are the most significant ones in the sense that any variation in these coefficients would introduce large changes in the model response. The coefficients that most influence the dynamics of the UAV are found by fixing all coefficients in equation (6) at their average values, while in turn varying each coefficient over its entire range. The frequency and time responses of the model are then analysed at various trim velocities to determine how a particular coefficient influences the dynamic characteristics. Proceeding in this way, it is determined that the coefficients $X_{q}, Z_{\theta}, Z_{q}, M_{u}, Z_{\delta \mathrm{e}}$, and $M_{\delta \mathrm{e}}$ are the most significant ones in the sense that any variation in these coefficients would introduce large changes in the model response. This is expected, since any variation in $X_{q}$ and $Z_{\theta}$ mainly affects the phugoid damping and variation in $Z_{q}$ affects the damping and frequency of the phugoid mode. Variation in $M_{u}$ affects primarily the short period frequency and variations in $Z_{\delta \mathrm{e}}$ and $M_{\delta \mathrm{e}}$ mainly affect the amplitude of the dynamic response of the aircraft. Figures 9 to

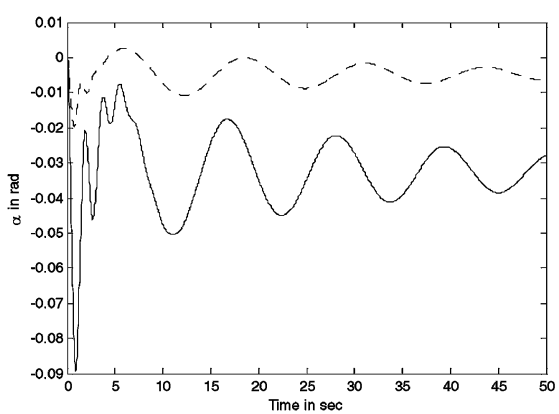



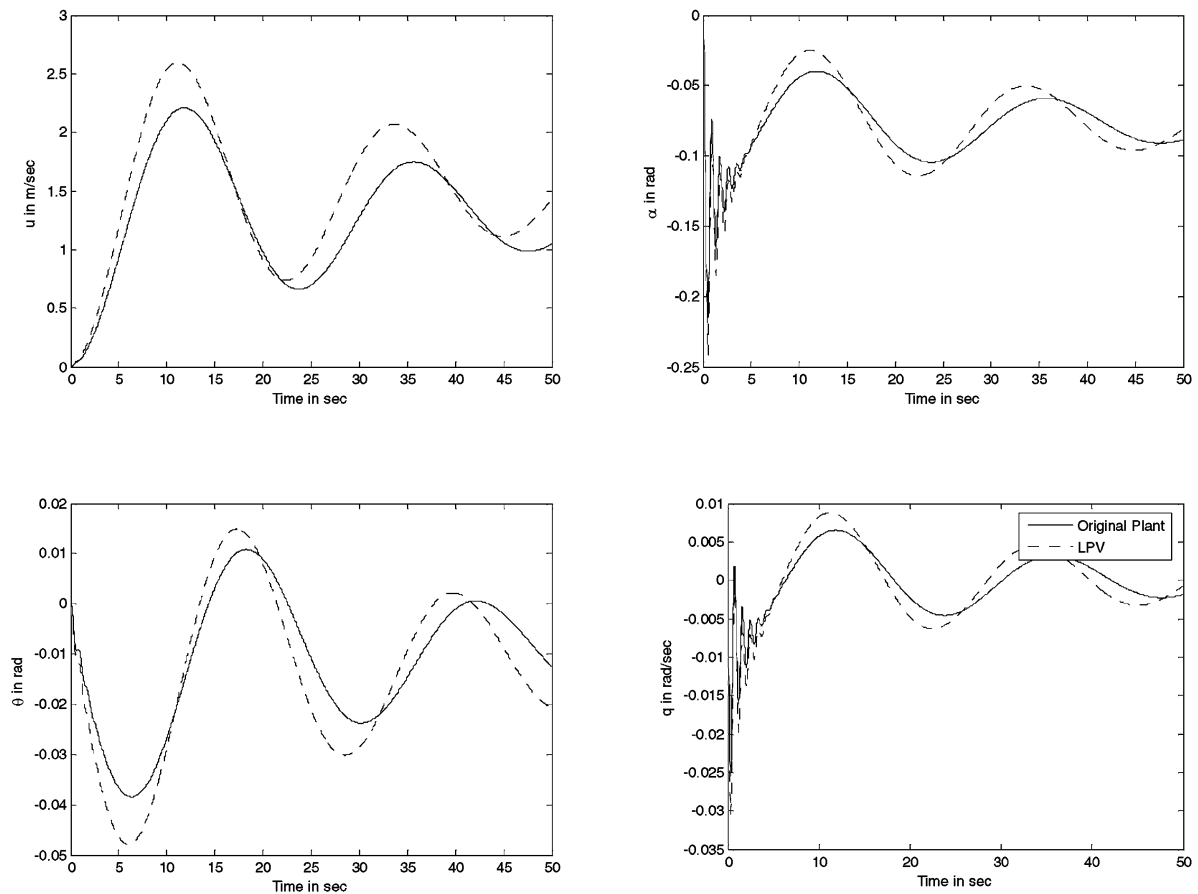

Fig. 10 Open-loop time responses of actual model and LPV model at $47 \mathrm{~m} / \mathrm{s}$
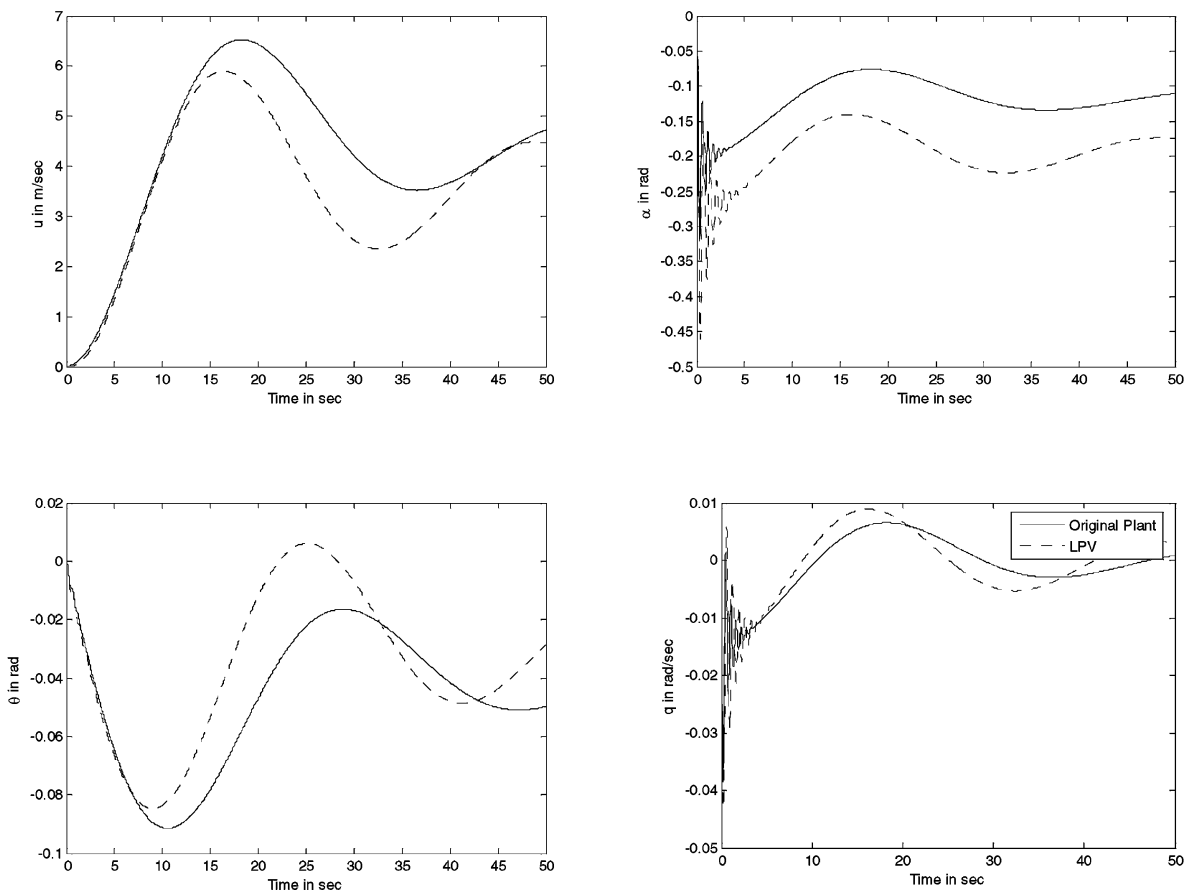

Fig. 11 Open-loop time responses of actual model and LPV model at $72 \mathrm{~m} / \mathrm{s}$

11 show the open-loop responses of original plant and LPV plant for a step FTV input at 22, 47, and $72 \mathrm{~m} / \mathrm{s}$. A comparison of the responses shows that the difference in the responses is high at $22 \mathrm{~m} / \mathrm{s}$ and $72 \mathrm{~m} / \mathrm{s}$, while there is good match at $47 \mathrm{~m} / \mathrm{s}$ owing to the average value chosen for most coefficients in equation (6). Such differences notwithstanding, the simplified LPV model is chosen for controller design, since it makes the design of controller simpler.

All elements in $A(V)$ that appear as functions of $V$ are now considered as 'structured' uncertainties, represented by a single variable $\alpha,|\alpha| \leqslant 1$. Note that 
as $V$ varies from 22 to $72 \mathrm{~m} / \mathrm{s}, \alpha$ varies from -1 to 1 . Thus, $A(V)$ in equation (6) becomes

$$
\begin{aligned}
& A(V)=A_{0}+A_{1} V=A_{0}+47 A_{1}+25 \alpha \\
& {\left[\begin{array}{cccccccccc}
0 & 0 & 0 & 0 & 0 & 0 & 0 & 0 & 0 & 0 \\
0 & 0 & 0 & 0 & 0 & 0 & 0 & 0 & 0 & 0 \\
0 & 0 & 0 & 0 & 0 & 0 & 0 & 0 & 0 & 0 \\
0 & 0 & 0 & 0 & 0 & 0 & 0 & 0 & 0 & 0 \\
0 & 0 & 0 & 0 & 0 & 0 & 0 & A_{z}(5,8) & 0 & 0 \\
0 & 0 & 0 & 0 & 0 & 0 & A_{1}(6,7) & A_{1}(6,8) & A_{1}(6,9) & 0 \\
0 & 0 & 0 & 0 & 0 & 0 & 0 & 0 & 0 & 0 \\
0 & 0 & 0 & 0 & A_{1}(8,5) & 0 & 0 & 0 & A_{1}(8,9) & 0 \\
0 & 0 & 0 & 0 & 0 & 0 & 0 & 0 & 0 & 0 \\
0 & 0 & 0 & 0 & 0 & 0 & 0 & 0 & 0 & 0
\end{array}\right]}
\end{aligned}
$$

Also note that the plant matrix $\boldsymbol{P}$ is of polytopic nature, i.e. assuming the time-varying parameter $V$ can be expressed in terms of its vertices as $V=\alpha_{1} 22+\alpha_{2} 72$, with $\alpha_{1}, \alpha_{2} \geqslant 0$ and $\alpha_{1}+\alpha_{2}=1, P(V)$ can also be expressed in terms of its vertices as $P(V)=\alpha_{1} P(22)+\alpha_{2} P(72)$. The unstructured input multiplicative uncertainty $W \Delta_{1}$, with $W=\frac{4 s+6}{s+40}$ and $\left\|\Delta_{1}\right\|_{\infty} \leqslant 1$ in Fig. 8 helps in incorporating uncertainties that arise from the approximation of the stability and control derivatives in $A(V)$ with their average values. $M$ is the matching model, which the closedloop system is expected to match. The reference model $M$ is chosen to be of diagonal form to achieve decoupling between height and total speed responses

$$
M=\left[\begin{array}{cc}
0 & 0 \\
\frac{100}{s^{2}+20 s+100} & 0 \\
0 & \frac{100}{s^{2}+20 s+100}
\end{array}\right]
$$

A damping ratio of 1 and undamped natural frequency of $10 \mathrm{rad} / \mathrm{s}$ are chosen for the matching model. Unity d.c. gain is required for zero steady state error to step inputs, while damping and frequency of $M$ are dictated by the level 1 handling quality requirements for full-scale aircraft. It is to be noted that such handling quality requirements are taken as benchmark in the absence of any specifications for the DEMON UAV. $P(V)$ is the plant model which varies linearly with trim velocity $V . w_{\mathrm{g}}$ is the vertical gust obtained as the output of the Dryden model (the input to which is white noise). $d$ is the output disturbance. The choice of weights $W_{1}$ and $W_{2}$ is problem dependent and requires elaborate tuning to achieve prescribed closed-loop specifications. The general rules for choosing the weighting functions are outlined below. The reference inputs and gust disturbances are mainly composed of lowfrequency signals. As a result, disturbance rejection and matching of outputs to reference inputs can be achieved by choosing a low pass filter for $W_{1}$. On the contrary, sensor noise and plant uncertainties are high-frequency phenomena, and high pass filters are chosen for $W_{2}$ to achieve sensor noise rejection and robustness. The final choice of $W_{1}$ and $W_{2}$ are

$$
\begin{aligned}
& W_{1}=\left[\begin{array}{ccc}
\frac{(80 s+1)}{\left(s^{2}+20 s+1\right)} & 0 & 0 \\
0 & \frac{0.031(\mathrm{~s}+1000)}{(\mathrm{s}+14)} & 0 \\
0 & 0 & \frac{0.018(\mathrm{~s}+1000)}{(\mathrm{s}+14)}
\end{array}\right] \\
& W_{2}=\left[\begin{array}{cc}
\frac{(0.1 s+1)}{(s+90)} & 0 \\
0 & \frac{(0.2 s+1)}{(s+90)}
\end{array}\right]
\end{aligned}
$$

A band-filter is chosen for the pitch rate channel in $W_{1}$, for rejection of disturbance and cross-coupling effects on the pitch rate response. The low pass filters on the height and total velocity channels in $W_{1}$ reflect the requirement of keeping the error between closed-loop and matching model responses low at low frequencies. In contrast, the weights on the FTV and throttle actuator channels are high pass in order to allow low-frequency control effort.

As the LPV system considered in this paper is polytopic, the LPV controller can also be chosen as a polytopic controller of the form

$$
K_{\mathrm{f}}(V)=\alpha_{1} K_{1}+\alpha_{2} K_{2}
$$

where $K_{1}$ and $K_{2}$ are the controllers designed at the vertices of the velocity polytope, i.e. at 22 and $72 \mathrm{~m} /$ s. $\alpha_{1}$ and $\alpha_{2}$ are the solutions of the convex decomposition problem

$$
V=\alpha_{1} 22+\alpha_{2} 72
$$

or in the present case, $K_{\mathrm{f}}(V)=K_{1}\left(1-\alpha_{2}\right)+\alpha_{2} K_{2}=$ $K_{1}\left(\frac{1-\alpha}{2}\right)+K_{2}\left(\frac{1+\alpha}{2}\right)$. In fact, this polytopic nature of the plant and controller is exploited in literature to reduce infinite number of constraints imposed by the LMIs arising out of the quadratic $H_{\infty}$ performance condition to a finite set of LMIs (e.g. reference [16]). However, the complexity of the solution of the LMIs is a disadvantage. In this paper, $\mu$ synthesis is used for the design of LPV polytopic controllers that can be described by equation (7). In order to express the frequency requirements described above in a more concise form, the closed- 
loop system in Fig. 8 is recast in the form of Fig. 12. The generalized plant $G$ now contains the plant $P$ along with the various weighting functions in Fig. 8. $\Delta$ represents the uncertainty, collected together from the closed-loop interconnection in Fig. 10 and has the form $\Delta=\operatorname{diag}\left(\alpha I_{11}, \Delta_{\mathrm{i}}\right)$. The closed-loop system in Fig. 12 is said to be stable and satisfy robust performance if and only if $\left\|F_{\mathrm{u}}\left(F_{1}(G, K), \Delta\right)\right\|_{\infty}<1$, for all $\|\Delta\|_{\infty} \leqslant 1$.

$K$ is thus the controller designed to satisfy the performance specification $\left\|T_{z w}\right\|_{\infty}<1$, where $T_{z w}$ is

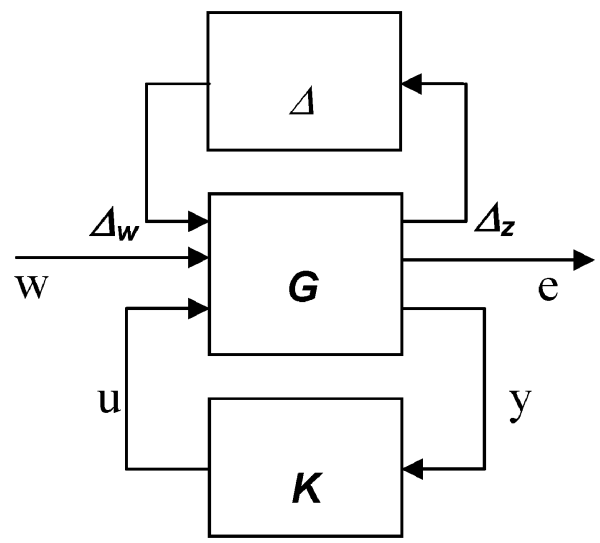

Fig. 12 Representation of the uncertain plant under feedback the closed-loop transfer function from $w=\left\lfloor\begin{array}{ll}r & w_{\mathrm{g}}\end{array}\right\rfloor^{\prime}$ to the performance outputs $z=\left[\begin{array}{ll}z_{1} & z_{2}\end{array}\right]^{\prime}$ along all variations of $V$.

\section{CLOSED-LOOP ANALYSIS}

The controller $K$ designed using Robust Control Toolbox v6.2 in MATLAB ${ }^{\circledR} \mathrm{v} 7.0$, is composed of two constituent controllers, namely $K_{1}$ obtained by making $\alpha=-1$ and $K_{2}$ obtained by making $\alpha=1$. The robustness of the controller to perturbations is assessed using $\mu$ bounds. The controller guarantees robust performance for the closed-loop system in Fig. 12 if and only if the following condition is satisfied sup $\mu_{\Delta k}(M(j \omega))<1$, where $\Delta_{k}:=\operatorname{diag}[\Delta$, $\omega \in[0, \infty]$

$\left.\Delta_{o}\right], \Delta_{o} \in C^{m \times p}, m$ and $p$ being the dimensions of $w$ and $e$ respectively and $M$ is the transfer function from $\left[\begin{array}{ll}\Delta_{w} & w\end{array}\right]^{\prime}$ to $\left[\begin{array}{ll}\Delta_{z} & e\end{array}\right]^{\prime}$. The controller guarantees robust stability for the closed-loop system if and only if the condition $\sup _{\Delta} \mu_{\Delta}\left(M_{11}(j \omega)\right)<1 \quad$ (the reader is referred to reference [22] for a detailed treatment of $\mu$ analysis. Figures 13 and 14 show the $\mu$ bounds for robust stability and performance respectively. As can be seen the maximum value of upper $\mu$ bound in Fig. 13 is 0.79 , which proves that

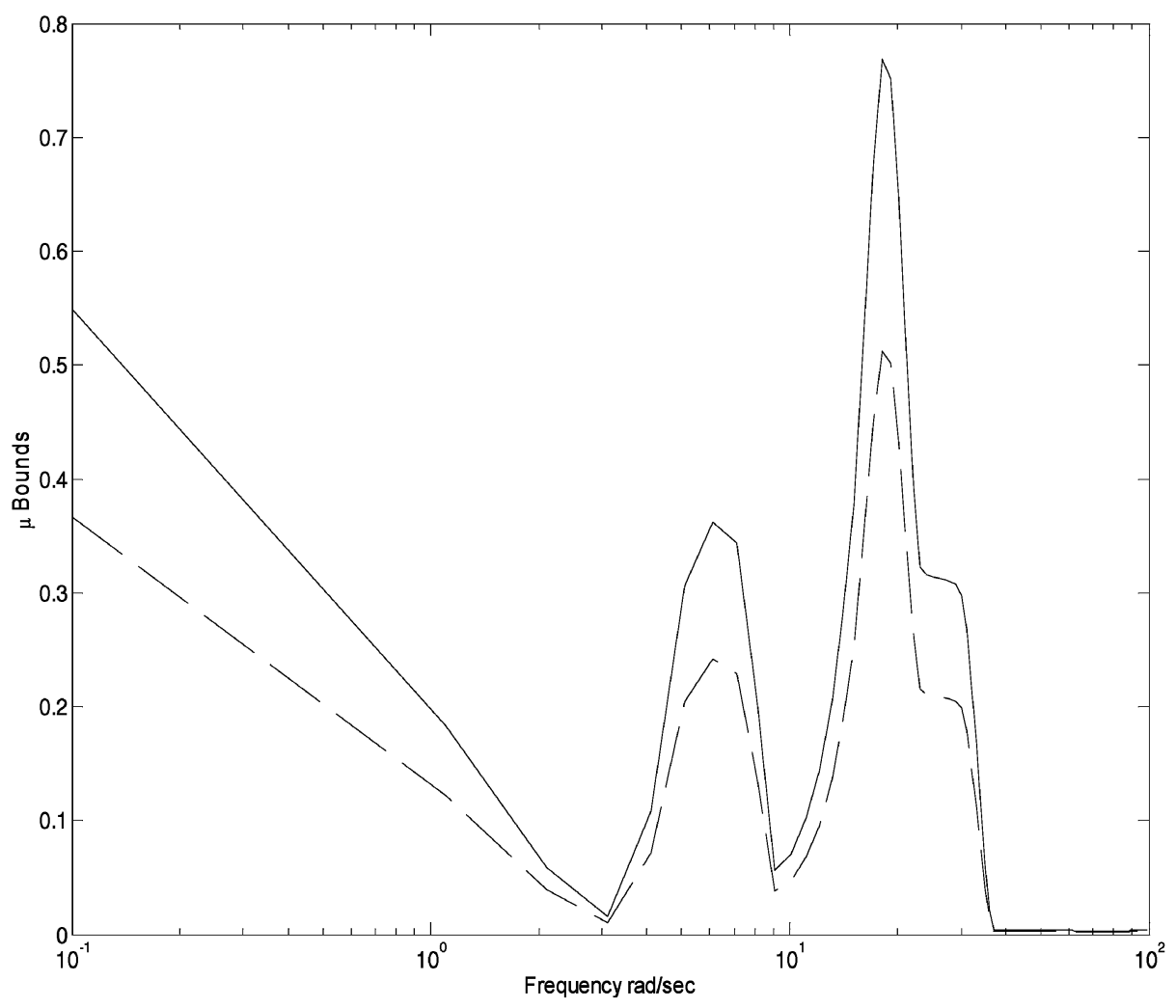

Fig. $13 \mu$ bounds for robust stability 


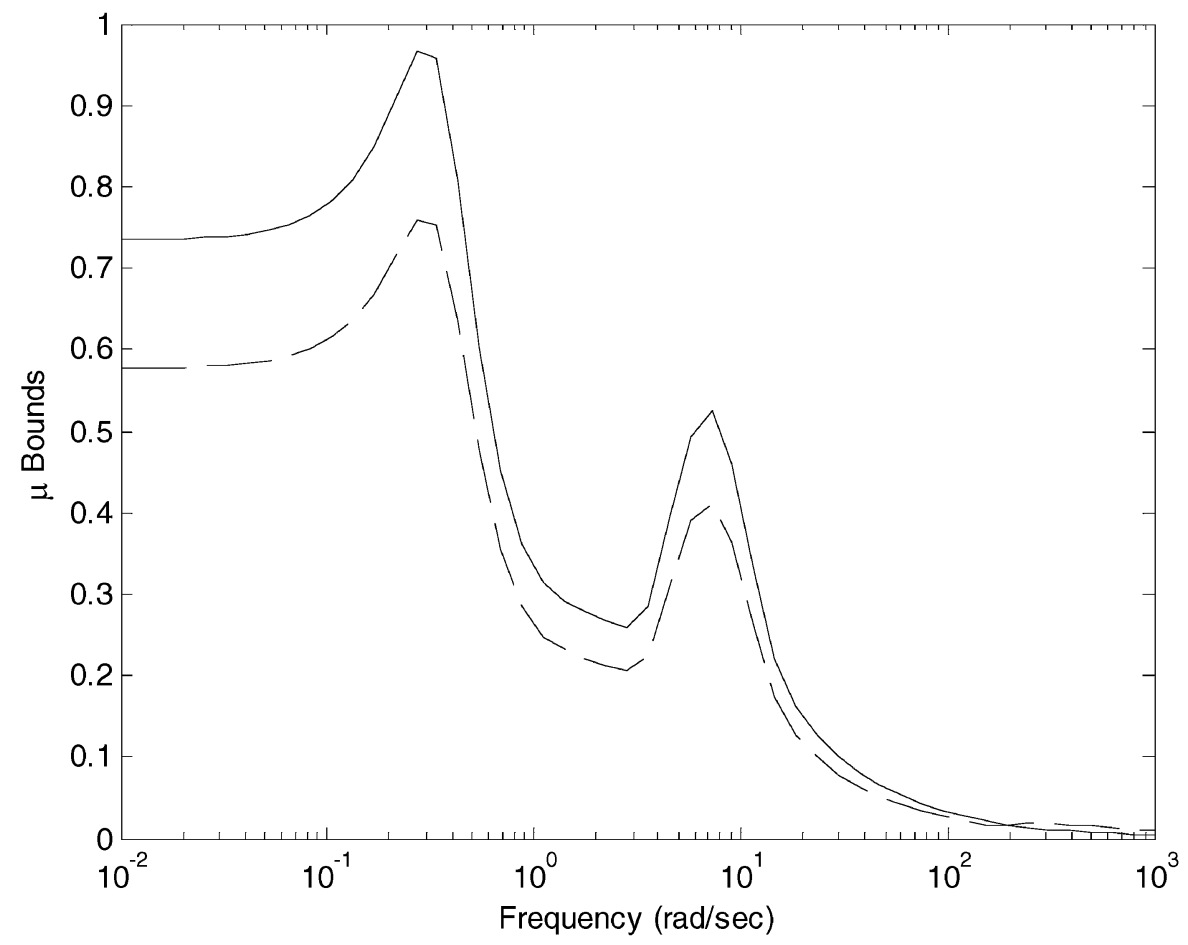

Fig. $14 \mu$ bounds for robust performance
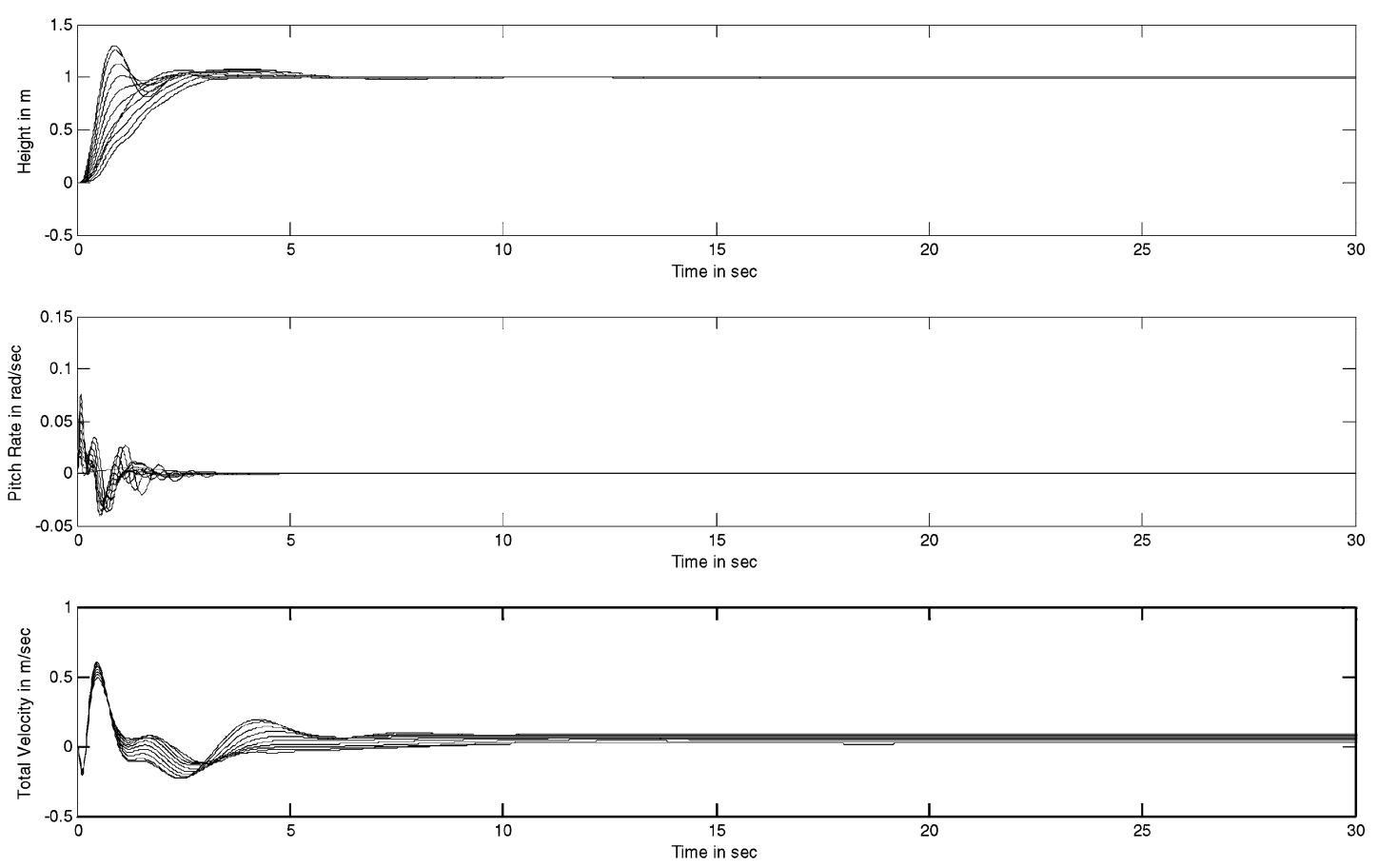

Fig. 15 Step response to a unit height demand

the closed-loop system is robustly stable for all variations in dimensional derivatives. The condition for robust performance is also satisfied since the maximum $\mu$ bound in Fig. 14 is 0.97 . Although this value is high, such a compromise in robustness can lead to superior closed-loop performance. Also, since the $\mu$ bounds are calculated with the augmented controller $K$, the robustness of individual con- 
trollers $K_{1}$ and $K_{2}$ are guaranteed automatically. In fact, this can also be seen in the closed-loop responses to a unit step demand in the height channel, shown in Fig. 15. The steady state error is negligible and represents marked improvement with the slow responses of Figs 9, 10, and 11. It should also be noted that the level of decoupling between height and total velocity responses, as measured from the ratio of total velocity to height, varies only marginally from 0.46 at $22 \mathrm{~m} / \mathrm{s}$ to 0.5 at $72 \mathrm{~m} / \mathrm{s}$. Such consistency in decoupling is particularly important for a remotely operated UAV since change in height should not impact the speed of the vehicle. Also, the settling time on all the output channels is of the order of $5 \mathrm{~s}$, which indicates that the controller with FTV and throttle inputs is successful in achieving good handling qualities.

\section{CONCLUSIONS}

Mathematical models of FTV actuators have been derived using experimental data. The models were then used in the design of a flight control system for the longitudinal dynamics. It was shown that $\mu$ synthesis can be used to design robust LPV controllers by representing the plant in an LFT form. The LPV controller designed using FTV and the throttle as control inputs resulted in satisfactory closed-loop performance with guaranteed stability over the entire cruise speed range. The proposed approach is considerably simpler than existing techniques for LPV controller design and can prove useful for the practising flight control engineer. A comparative performance analysis of both FTV and CC illustrated the slight superiority of conventional control surfaces. This can, however, be rectified through the use of powerful onboard sources of secondary jet and remains an area of future research.

\section{REFERENCES}

1 Alcorn, C. W., Croom, M. A., Francis, M. S., and Ross, H. The X-31 aircraft: advances in aircraft agility and performance. Progress in Aerospace Sciences, 1996, 32, 377-413.

2 Bowers, A. and Pahle, J. Thrust vectoring on the NASA F-18 high alpha research vehicle. NASA TM4771, 1996.

3 Vickers, J. Propulsion analysis of the F-16 multiaxis thrust vectoring aircraft. Fourth High Alpha Conference, Edwards, CA, NASA CP-10143, 1994.

4 Friehmelt, H. Thrust vectoring and tailless aircraft design - review and outlook. AIAA paper 96-3412, 1996.
5 Berens, T. M. and Bissinger, N. C. Thrust vector behavior of highly integrated asymmetric nozzles for advanced fighter aircraft. AIAA paper 98-0948, 1998.

6 Panitz, T. and Wasan, D. T. Flow attachment to solid surfaces: the Coanda effect. AIChE Journal, 1972, 18(1), 51-57.

7 Wing, D. Static investigation of two fluidic thrust vectoring concepts on a two dimensional convergent divergent nozzle. NASA Technical Memorandum 4574, December 1994.

8 Miller, D. N., Yagle, P. J., and Hamstra, J. W. Fluidic throat skewing for thrust vectoring in fixedgeometry nozzles. AIAA paper 1999-365, 1999.

9 Flamm, J. D. Experimental study of a nozzle using fluidic counterflow for thrust vectoring. AIAA paper 98-3255, 1998.

10 Banazadeh, A., Saghafi, F., Ghoreyshi, M., and Pilidis, P. Multi-directional co-flow fluidic thrust vectoring intended for a small gas turbine. AIAA paper 2007-2940, 2007.

11 Mason, M. S. and Crowther, W. J. Fluidic thrust vectoring for low observable air vehicles. AIAA paper 2004-2210, 2004.

12 Skogestad, S. and Postlethwaite, I. Multivariable feedback control: analysis and design, 2005 (John Wiley, London).

13 Nichols, R. A., Reichert, R. T., and Rugh, W. J. Gain scheduling for $H_{\infty}$ controllers: a flight control example. IEEE Trans. Autom. Control, 1993, 1(2), 69-79.

14 Packard, A. Gain-scheduling via linear fractional transformations. System Control Lett., 1994, 22, 79-92.

15 Packard, A. and Becker, G. Quadratic stabilization of parametrically-dependent linear systems using parametrically-dependent linear, dynamic feedback. In Proceedings of the Technical Sessions on Advances in robust and nonlinear control systems, ASME Winter Annual Meeting, Anaheim, USA, 8-13 Nov. 1992, pp. 29-36.

16 Apkarian, P., Gahinet, P., and Becker, G. Selfscheduled $H_{\infty}$ control of linear parameter-varying systems: a design example. Automatica, 1995, 31, 1251-1261.

17 Wang, F. and Balakrishnan, V. Improved stability analysis and gain-scheduled controller synthesis for parameter-dependent systems. IEEE Trans. Autom. Control, 2002, 47, 720-734.

18 Wu, F. and Dong, K. Gain-scheduled control of LFT systems using parameter-dependent Lyapunov functions. Automatica, 2006, 42, 39-50.

19 Balas, G., Fiahlo, I., Packard, A., Refrow, J., and Mullaney, C. On the design of the LPV controllers for the F-14 aircraft lateral-directional axis during powered approach. In Proceedings of American Control Conference, 1997, pp. 123-127.

20 Marocs, A. and Balas, G. Development of linearparameter varying models for aircraft. J. Guidance, Control Dynamics, 2004, 27, 218-228. 
21 Mason, M. S. and Crowther, W. J. Fluidic thrust vectoring for low observable air vehicles. University of Manchester Report.
22 Packard, A. and Doyle, J. The complex structured singular value. Automatica, 1992, 29(1), 71-109. 